\title{
Validity of the Dialog Preference Scale for Nurses' Communication with Older People in Japan
}

Hiroko Shimizu

Department of Chronic Adult Nursing, School of Nursing, Faculty of Medicine, Kagawa University, Japan

\begin{abstract}
This study examines the validity for applying nurses of the Dialogue Preference scale for elderly people created from data on nursing students in Japan. The design was a forward-looking quantitative questionnairebased study. Data collection was conducted at hospitals and seminars in Japan. Participants were nurses ( $\mathrm{n}$ = 186; males: $2.7 \%$; females: $97.3 \%$ ) and nursing students $[\mathrm{n}=552$; males: $2.0 \%$ (38.4 \pm 10.3 years); females: $98.0 \%$ (22.9 \pm 4.9 years)]. Nursing student data from a study with the same questionnaire were obtained retrospectively. Data were gathered using the 15-item Shimizu Scale, which measures attributes and four subconcepts (bewilderment, anxiety, cognitive bias, and communication difficulty). Higher scores indicate more negative cognitions or attitudes (maximum score: 28). Data were descriptively analyzed using t-tests and secondary factor analysis of confirmatory factors. The responses of nurses were compared with the previously collected responses of nursing students who differed from nurses in their experience and knowledge. Nurses' total scores (mean: 3.94$)$ were significantly lower than the nursing students' scores (mean: 4.4), indicating that nurses had a more positive view of communication with older people than nursing students. Moreover, a significant difference in the anxiety scores was observed between nurses with $<1$ year of experience and those with more experience. Experienced nurses had a more positive attitude regarding communication with older people compared with nursing students. Since this result instructed the current research results, the Shimizu Scale is appropriate for nurses.
\end{abstract}

\section{Background and Purpose}

In this study, the quality of involvement was operationally defined as dialog preference. A previous report stated that knowledge can be transformed into a positive attitude in nursing students and emphasized the need for an education program that considers cultural background [1]. Cultural features are a key part of this knowledge base. Education levels can also affect attitudes and communication among nurses. Moreover, nurses' gerontological knowledge and understanding strongly influence their attitudes toward older people. Those with higher levels of education tend to exhibit more positive attitudes and perceptions. This implies that the provision of adequate and culturally sensitive education to nurses must be ensured.

This research has developed from the realities of Japan that has become an aging society. As of 2012, the census data indicate that the total population of Japan is declining after peaking in 2010 . Simultaneously, the percentage of older people within the total population is steadily increasing. In $2018,28.2 \%$ of the total Japanese population was classified as elders. This percentage is projected to increase to $33.3 \%$ by 2036 and $38.4 \%$ by 2065 based on the 2017 estimates [2]. The problems faced by Japan's aging population should be addressed in policy measures designed to ensure that older people can live healthy and independent lives. Thus, the Healthy Japan 21 policy was released in 2000, followed by the Health Promotion Law in 2002 .

Before the 1980s, aging was viewed as a period of increasing physical and mental frailty. However, this viewpoint has clearly changed since the early 1990s and has been influenced by the notion of "successful aging" advocated by Rowe and Kahn [3]. As expressed in the terminal decline model proposed by psychologists, crystalized intelligence continues to function until just before death, and older people are capable of contributing to society, which should be considered a desirable objective [4]. Nurses involved in healthcare with older people should understand the characteristics of aging and be free of cognitive bias when treating older people [5].

\section{Publication History:}

Received: March 11, 2020

Accepted: April 06, 2020

Published: April 08, 2020

\section{Keywords:}

Ageism, Attitude, Bias, Elderly, Nursing students, Communication form of discrimination, such as racism and sexism. Specifically, Butler [6] defined ageism as "a process of systematic stereotyping and discrimination against people because they are old." This type of bias, which results from cultural and social influences [7], can be difficult to correct through education. Indeed, this may be a specific problem in Japan, with Palmore [7] observing that the general Japanese population holds feelings of respect and contempt for older people. In February and March 2004, the Japanese Cabinet Office performed the "Survey on Public Attitudes to Aging," which used the Fraboni Scale of Ageism (FSA) to evaluate cognitive bias against older people among 6,000 Japanese respondents aged $\geq 20$ years. Respondents who were older males living in major cities or prone to illness expressed strong feelings of avoidance and rejection toward older people. People form views about others beginning from their childhood. Another research in Japan indicated that views toward older people are positive in elementary school but less positive in junior high school and high school. Children frequently consider older people to be physically weak [8]. Therefore, Japanese culture and social influences may enhance the prejudice against older people.

These cognitive biases are present in the general population. However, Japanese nursing students who have been educated about older people tend to view older people as more "active," "considerate," "individual," "worthy of respect," and "expressive," thus exhibiting attitudes that are not necessarily negative [9]. Nursing students are more likely to understand the physical and psychosocial

"Corresponding Author: Prof. Hiroko Shimizu, Department of Chronic Adult Nursing, School of Nursing, Faculty of Medicine, Kagawa University, Japan, Tel/ Fax: +81-87-891-2240; E-mail: hshimizu@med.kagawa-u.ac.jp

Citation: Shimizu H (2020) Validity of the Dialog Preference Scale for Nurses Communication with Older People in Japan. Int J Nurs Clin Pract 7: 322. doi https://doi.org/10.15344/2394-4978/2020/322

Copyright: (C) 2020 Shimizu. This is an open-access article distributed under the terms of the Creative Commons Attribution License, which permits unrestricted use, distribution, and reproduction in any medium, provided the original author and source are credited. 
changes experienced by older people because of their exposure to gerontological education and clinical training, presumably allowing them to treat older people appropriately and with respect. In other words, irrespective of their original cognitive biases, nursing students should acquire positive views of older people through their training. Thus, improving the thoughts and attitudes of nursing students can help improve care for older people with nurses.

Despite this expectation, it was reported that nurses tend to exhibit ageism similar to the general population [10], potentially compromising the provision of care. A study on ageism among medical staff members found that there were extremely negative attitudes toward older people among those who were frequently involved with providing care to severely disabled older patients [10]. Such negative attitudes can significantly affect the quality of care. Nurses should have positive attitudes if they are to provide effective healthcare. Conversely, older people do not often shy away from young people and caregivers. Instead, they often want to remain involved or hear and tell stories [11]. In other words, lively dialogs are important for providing care to older people. Even if a nurse holds a negative bias against older people, his or her knowledge base should facilitate effective communication. In Albert Ellis' logical emotional therapy, he stated that it is possible to transform behavior that results from cognitive distortions. Therefore, it is necessary to evaluate the existing bias when nurses perform clinical tests. Several well-known tools help educators measure the attitudes of nurses toward older people.

The most commonly used scale for measuring cognitive perceptions of older people is the Attitude Scale for Older People $[8,12,13]$, which includes a negative indicator for ageism. The best-known scale for evaluating ageism is the FSA [12] revised by Rupp, Vodanovich, and Credé [14] into a 29-statement survey that measures cognitive bias against older people. Test statements are rated using a 4-point Likerttype scale. Scores range from 29 to 116 with a higher score indicating more ageism. FSA was developed in France but has been used in Israel, Australia, Turkey, and Japan [15,16]. A study was performed using this FSA in Australia by Leung et al., [15]. These results apply to nurses as well. Thus, improvement can be targeted if the problem can be identified and evaluated. FSA measures ageism among nursing students and evaluates trends in ageism based on age groups. However, FSA only evaluates cognitive bias against older people at a cognitive level and does not measure what occurs during conversations.

The Kogan Attitudes toward Old People Scale [13] measures the attitudes of nurses toward older individuals. This scale comprises 34 questions divided into equal groups of positively and negatively framed questions with answers given using a 6-point Likert-type scale (from strongly disagree to strongly agree). This scale is used as a training tool for nursing students [17] and healthcare workers [18]. Kogan's scale measures the influence of nurses' and nursing students' attitudes toward older people. Its efficacy has been evaluated in Sweden [19] and Israel [20]; however, item 28 is not suitable in the Japanese context. Item 28 is "Most old people seem quite clean and neat in their personal appearance" in Kogan's scale. Older people in Japan retire and lose their occupational role and become less concerned about their appearance. Dressing up for the sake of their jobs and not for themselves is their common characteristic [21]. In other words, older people tend to be apathetic about evaluating other people. This may make a young person feel that an older person is a difficult conversation partner. This implies that older people have a distinctive sense of individuality. Palmore [7] and Narita et al. [21] provided evidence of specific prejudice against older people in Japan, implying that it is necessary to evaluate nurses involved in the care of older Japanese people.

Shimizu [22] has developed a scale by which the dialog preference attitude of Japanese nursing students toward older people can be measured. It is the purpose of this study to examine whether it is useful for nurses.

\section{Methods}

\section{Study design}

This was a quantitative, questionnaire-based comparative study. The specific purpose of this study was to assess the validity of the data obtained of nurses using the Shimizu Scale questionnaires, which were developed with previously collected data from nursing students [23].

\section{Setting and sample}

This study was a cross-sectional questionnaire-based survey on nurses between 2009 and 2012. To minimize the potential for geographical bias, the study was performed in several places. Nurses from four investigation venues in Japan were included: those who attended dementia seminars in Osaka and Tokyo and nurses from one medium-sized hospital for work-related accidents in Shikoku. The nurses had applied freely and, thus, became random samples. It is known that nurses who care for older people with disabilities such as dementia have a bias toward older people [24]. The survey was targeted nurses who are prone to prejudice. All the respondent nurses are currently practicing clinical nursing. Nurses with cognitive impairment were excluded.

\section{Ethical consideration}

Ethics approval was obtained from the $\mathrm{K}$ University Ethics Committee (2014-059), and nurses who chose to participate provided consent and were not directly approached by the primary researcher. Instead, an assistant explained the study and distributed consent forms and questionnaires. Questionnaire responses were anonymous and did not record details of the respondents or their institution.

\section{Measurements/instruments}

The four sub-concepts of the Shimizu Scale are reliable [23]. These concepts relate to relationships with older people and include bewilderment shown through uncertainty, apprehension that is inherent in anxiety, cognitive bias associated with ageism, and difficulty in communication.

No other standardized scale is comparable to the Shimizu Scale. However, concurrent validity was detected for the "anxiety in relationships" item on the Shimizu Scale compared with the "authoritative anxiety" item within the Social Anxiety Scale in Social Situations [25]. The Shimizu Scale also demonstrated predictive validity for the targeted achievement level of nursing students' clinical training. The total coefficient of reliability $(\alpha)$ was 0.811 , thus, confirming internal consistency reliability. For the first (bewilderment), second (anxiety), third (cognitive bias), and fourth (communication difficulty) sub-factors, the coefficients of reliability (a) were $0.764,0.687,0.671$, and 0.670 , respectively. 
Citation: Shimizu H (2020) Validity of the Dialog Preference Scale for Nurses' Communication with Older People in Japan. Int J Nurs Clin Pract 7: 322. doi: https://doi.org/10.15344/2394-4978/2020/322

Page 3 of 6

\section{Data Collection/procedure}

In this study, the 15-item Shimizu Scale was used (Table 1). This scale collects details of base attributes and measures 15 key items (including anxieties, knowledge, behavior, and value systems) on a 7-point Likert-type scale with higher scores indicating more negative thoughts and attitudes related to the communication of the nurses with older people. Unlike other scales, the Shimizu Scale (Table 1) measures the attitudes of the caregivers and also considers their educational levels and respondents' cultural characteristics [22]. The scale's validity and reliability were confirmed on the basis of data collected from Japanese nursing students. These data were later confirmed in a population of nurses [22].

\section{Data analysis}

Data were analyzed as descriptive statistics. As appropriate, student's t-tests and secondary factor analyses of confirmatory factors were performed. The Amos 5 statistical analysis program was used for all analyses. Data are presented as mean (IBM, SPSS, standard deviation ver. 24) unless otherwise specified.

\section{Results}

In total, 230 nurses (6 males, 224 females) completed the survey. However, only 186 provided valid results. Nurses who attended dementia seminars in Osaka $(\mathrm{n}=53)$ and Tokyo $(\mathrm{n}=70)$ and who worked at a medium-sized hospital in Shikoku $(\mathrm{n}=63)$ were recruited. The mean age of nurses was $38.42(\mathrm{SD}=10.31)$ years, and their average years of nursing experience was $11.64(\mathrm{SD}=9.34)$ years. The comparison data set comprised 552 (males, $2 \%$; females, 98\%) nursing students with an average age of 23.09 years $(\mathrm{SD}=5.08)$. The data of 552 respondents were valid (i.e., without omissions)from a total of 833 collected responses (80 undergraduates, 236 junior college students, and 517 vocational students) in Tokyo [22]. The nurses who responded had experience of nursing senior citizens who had participated in a dementia seminar.

The mean item score on the Shimizu Scale was $3.94(\mathrm{SD}=11.68)$ for nurses. The average sub-factor score for nurses was $14.18(\mathrm{SD}=5.14)$ and for nursing students was $18.47(\mathrm{SD}=5.81)$ for bewilderment, $16.75(\mathrm{SD}=18.82)$ for anxiety, $13.67(\mathrm{SD}=14.41)$ for cognitive bias, and $14.45(\mathrm{SD}=14.36)$ for communication difficulty. There was no significant difference $(\mathrm{p}>0.1)$ in the responses of nurses from Osaka [56.62 $(\mathrm{SD}=12.18)]$, Tokyo [58.49 $(\mathrm{SD}=12.75)]$, and Shikoku [61.70 $(\mathrm{SD}=9.46)]$. Of all the respondents, $140(62 \%)$ reported the experience of living with older people; 47 (21\%) had no experience of living with older people; 32 (14\%) were currently living together; and 8 (3\%) lived together with older people in the past.

The standardized estimated path coefficients for each of the four sub-factors for student nurses were 0.84 for bewilderment, 0.54 for anxiety, 0.37 for bias, and 0.74 for communication difficulty. The goodness of fit index (GFI) was 0.95 ; the adjusted goodness of fit index

\begin{tabular}{|c|c|c|}
\hline \multicolumn{3}{|c|}{ 1. Sex ( ) } \\
\hline \multicolumn{3}{|c|}{ 2. Age ( ) } \\
\hline \multicolumn{3}{|c|}{ 3. Clinical experience (years) ( ) } \\
\hline \multicolumn{3}{|c|}{ 4. Have you experienced living with older people? (Presently yes/In the past yes/no) } \\
\hline \multicolumn{3}{|c|}{$\begin{array}{l}\text { Please respond to the questions using the following scale that assumes that the distance between numbers is equal. Circle one number }(1-7) \text { that } \\
\text { best represents your opinion: } 1 \text { (Strongly disagree) }-2-3-4 \text { (Neither agree nor disagree) }-5-6-7 \text { (Strongly agree). }\end{array}$} \\
\hline No & Questions & $\begin{array}{l}\text { Grading (6-point Likert-type) } \\
\text { 1:strongly disagree, } \\
\text { 7: strongly agree }\end{array}$ \\
\hline 1 & $\begin{array}{l}\text { I am aware of a major age gap with older clients and find it difficult to think of suitable topics of } \\
\text { conversation. }\end{array}$ & $1-2-3-4-5-6-7$ \\
\hline 2 & I am uncertain how seriously I should listen to older clients. & $1-2-3-4-5-6-7$ \\
\hline 3 & $\begin{array}{l}\text { I always try to maintain a loud voice when I talk to older clients, so I tend to say things that are not really } \\
\text { what I want to say. }\end{array}$ & $1-2-3-4-5-6-7$ \\
\hline 4 & I do not know how to react when older clients repeat themselves. & $1-2-3-4-5-6-7$ \\
\hline 5 & $\begin{array}{l}\text { In conversation, when older clients talk about history, I am not sure what to do because my knowledge is } \\
\text { limited. }\end{array}$ & $1-2-3-4-5-6-7$ \\
\hline 6 & I worry about the vision of older clients. How do they see and how much do they see? & $1-2-3-4-5-6-7$ \\
\hline 7 & When talking to older clients, I wonder whether my voice is the right volume and if my tone is appropriate. & $1-2-3-4-5-6-7$ \\
\hline 8 & When talking to older clients, I worry that I may exhaust them. & $1-2-3-4-5-6-7$ \\
\hline 9 & I wonder how older clients view us, the young generation. & $1-2-3-4-5-6-7$ \\
\hline 10 & Older clients just want someone to listen to them all the time. & $1-2-3-4-5-6-7$ \\
\hline 11 & Older clients want somebody to listen to them and they want to talk about themselves. & $1-2-3-4-5-6-7$ \\
\hline 12 & It seems that once older clients start talking they often do not stop. & $1-2-3-4-5-6-7$ \\
\hline 13 & It is difficult to know how to speak to and deal with older clients who have dementia. & $1-2-3-4-5-6-7$ \\
\hline 14 & I find it hard to hold conversations with older clients with hearing difficulties. & $1-2-3-4-5-6-7$ \\
\hline 15 & I find it hard to communicate with older clients with total aphasia. & $1-2-3-4-5-6-7$ \\
\hline
\end{tabular}


(AGFI) was 0.93; the comparative fit index (CFI) was 0.93; and the root mean square error of approximation was 0.04 , thus confirming construct validity.

The path coefficients for dialog preferences in the sub-factor for nurses were 0.48 for bewilderment, 0.36 for anxiety, 0.34 for cognitive bias, and 0.72 for communication difficulty (Figure 1). When comparing the fit of the sub-concept analysis model, GFI was 0.91 (0.95 for nursing students); AGFI was 0.88 (0.93); and CFI was 0.94 (0.94) for nurses, indicating good model fit for nurses. However, a significant difference was seen in the apprehension score for nurses with $<1$ year experience compared with those with more experience ( $\mathrm{p}<0.05)$. Overall, compared with the nursing student data previously measured using the same scale [22], experienced nurses had more positive attitudes toward older people on the Shimizu Scale.

\section{Discussion}

Bewilderment (sub-factor 1) or relationship uncertainty may occur because of a large age gap. It can manifest as difficulty in talking, the need to repeat statements, and feeling at a loss to understand an older individual's personal history. Such intergenerational discord was observed in previous studies $[7,26]$. However, in this study, issues related to conversation, conversation methods, and differences in experiences as particular problems were identified. It is a reasonable result that experienced nurses have less confusion.

Conversational proficiency is acquired through experience; however, younger nurses can benefit from receiving age-specific communication training that can improve their communication with older people. After acquiring this knowledge, nursing students can put it into practice using micro skills [27]. A deeper understanding is usually possible for experienced nurses. However, for young nurses who have little experience, deeper understanding is possible only if they undergo training with respect to older people. Deepened understanding improves experience, and as our results [27] have demonstrated, it increases positive attitudes. Understanding older people is the only significant predictor of attitude and the basis for proven statistical causality [28]. The greater knowledge levels among nurses compared with nursing students indicate why the confusion score was low in the nurse group [1]. The communication between nurses and older people is affected by age, sex, and subjective health outlook [29]. However, this study did not consider sex-based differences.

The mean score for sub-factor 2, representing relationship anxiety or apprehension, was lower in nurses (16.75) than in nursing students

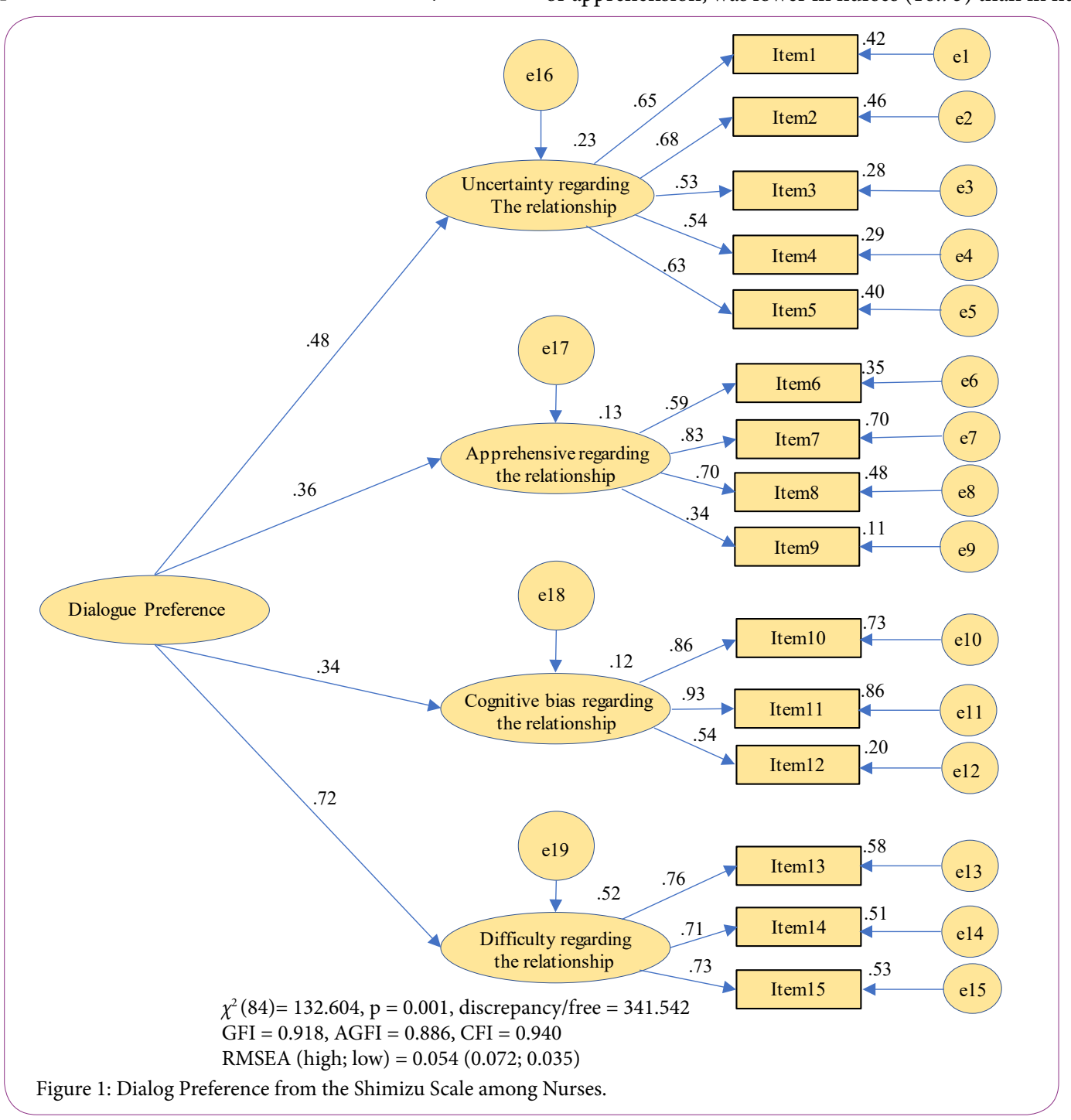


(18.82). This factor includes concern for an older person's eyesight, whether the volume and tone of the nurse's voice is appropriate, if the nurse is fatiguing the client, and curiosity about what older people think about the younger generation. Similarly, the nurses' knowledge and experience with sensory organs and corporeal frailty may have influenced this result. Additionally, the mean age among nurses was 38.39 years and that of nursing students was 21.09. Lowering this score may prevent one from developing anxiety that an older person has negative views about the younger generation. On comparing the education levels and wealth of older individuals with those of younger nurses, it was found that the disparities observed depended on their respective backgrounds [30].

The difference between the results of nursing students and nurses in the bewilderment (sub-factor 1) and anxiety (sub-factor 2) measures is related to the level of knowledge and experience about older people, which is supported by previous research. Based on survey results, King et al. [1] stated that to improve nurses' attitudes toward aging, knowledge regarding aging must be improved as a basic professional skill. Moreover, considering the ethnic differences reported in this study, additional efforts should be made to tailor culturally appropriate educational programs to nurses from different backgrounds. Such culturally appropriate educational programs can be assessed using the Shimizu Scale, which considers cultural background. In other words, such programs can assess whether nurses and nursing students with aggressive attitudes toward older people retain those attitudes after receiving targeted education.

The mean score for anxiety (sub-factor 2) was particularly high among nurses with $<1$ year of experience, indicating that while novice nurses are motivated to provide care, there is latent anxiety. Although novice nurses' skills are not fully developed, they want to provide an idolized form of nursing that they learn as students. Therefore, although motivation to provide care is high, they often lack confidence. Accordingly, it is vital that novice nurses be provided with dedicated education programs that offer instruction on how to take care of older people during their firstyear of training. If the patient's age makes the relationship between the nurse and patient difficult, it is pertinent that new nurses receive education to deal with this issue.

Our study also demonstrated that nurses scored lower (13.67) on sub-concept 3 (cognitive bias) compared with nursing students (14.41). This bias, or ageism, is influenced by socio cultural factors. Ageism involves stereotyping and prejudice against individuals or groups because of their age; the term was coined in 1969 by US gerontologist Robert Butler to describe discrimination against seniors [5]. Although the term ageism may be rather strong, especially given its association with sexism and racism, it appears to have been accepted by young nursing researchers in the USA [26]. In terms of conversation, ageism can manifest as thoughts that the older people want others to listen to them all the time or that they want to talk about themselves. Both nurses and nursing students revealed cognitive bias in this study, but nursing students lacked knowledge and experience, and their perceptions were more likely to be displayed in their language choice and attitudes. Following the completion of gerontological nursing practical training (training in conversational skills), the attitude of nursing students toward older people improved [31]. Thus, a person's attitude toward older people can improve if they acquire conversational skills and an understanding of the aging process. Prejudice involves stereotypical thinking and, therefore, requires deliberate education to effect change. Gerontological education helps encourage positive attitudes [32].
The scores for communication difficulty (sub-factor 4) did not differ between nurses (14.45) and nursing students (14.36). For these items, patients with communication disorders such as dementia (item 13), hearing impairment (item 14), or aphasia (item 15) were considered. Cognitive bias (sub-factor 3) and communication difficulty (sub-factor 4) were more positive as nursing experience increased. Although nurses have basic knowledge of nursing patients with communication disabilities, successful communication with such patients requires experience in this regard. The evaluation scale can help accumulate the success of such an experience. When nurses are educated, it is necessary to assess their learning. The Shimizu Scale could help to predict experimental factors based on observations of behaviors and attitudes of nurses.

Recruitment of nurses through specialized research forums may have resulted in a different outcome than that of ordinary nurses. It is likely that nurses who attend seminars on dementia have positive attitudes about older people. This limits the generalizability of the findings. Further studies with different groups of nurses are required. Particularly, an international comparison study could assist in the development of training methods that incorporate cultural differences.

\section{Conclusion}

Participants from differing geographies contribute to the strength of a study. In this study, the nurses exhibited lower bewilderment and anxiety, indicating lower prejudice compared with nursing students. It is likely that this was attributable to increased experience and knowledge among the nurses. It can be said that it is a reasonable result. Consistent with a previous report [22], the Shimizu Scale predicted these issues. From the above, the Shimizu Scale developed by nursing students has produced appropriate results in nurses who understand dementia as well as others. Using this scale, the characteristics of ageism among Japanese nurses can be revealed, which in turn can reveal challenges in education. It may be used in countries with similar backgrounds as Japan.

\section{Implications for Practice}

Quality nursing care requires reflective practice. The Shimizu Scale is a useful tool to help nurses and nurse educators to measure attitudes about the aging process. In turn, this can increase self-awareness and can be used as a basis from which communication skills can be improved and anxieties understood. Further empirical studies that target educational outcomes in such areas are warranted. The impact of gender differences, in particular, should be addressed in future research.

\section{Acknowledgments}

The author would like to thank Enago (www.enago.jp) for English language review.

\section{Competing Interests}

The author declare that there is no competing interests regarding the publication of this article.

\section{References}

1. King BJ, Roberts TJ, Bowers BJ (2013) Nursing student attitudes toward and preferences for working with older adults. Gerontol Geriatr Educ 34: 272291. 
2. National Institute of Population and Social Security Research (2017) Population Projections for Japan: 2016-2065

3. Rowe JW, Kahn RL (1987) Human aging: Usual and successful. Science 237: 143-149.

4. Karasawa A (1989) Mental functioning of older people. In M Tomonaga \& A Sato (Eds.). Aging of the brain and nervous system.Tokyo: Asakura Shoten.

5. Butler RN (1969) Age-ism: Another form of bigotry. Gerontologist 9: 243246.

6. Butler RN (1995) Ageism. In GL Maddox (Ed.), The Encyclopaedia of Aging. New York, NY: Springer.

7. Palmore EB (1999) Types of ageism. In EB Palmore (Ed.), Ageism: Negative and Positive. New York, NY: Springer Publishing Company, Inc.

8. Baba J (1993) Old man view of junior high school students-Measurement using old man view scale. Social Gerontology 38: 3-12.

9. Konnya, E., Takaoka, T., Fukazawa, K., \&Watanabe, T. (2009) An examination of nursing students' outlook toward older people after having visited daycare centers for seniors. Bulletin of Nayoro City University 3: 39-47.

10. Bernard M (1998) Backs to the future? Reflections on women, ageing and nursing. J Adv Nurs 27: 633-640.

11. Erikson EH, Erikson JM, Kivnick HQ (1986) Vital involvement, vital involvement in old age. New York, NY: WW Norton \& Company.

12. Fraboni $M$, Saltstone $R$, Hughes $S$ (1990) The Fraboni scale of ageism (FSA): An attempt at a more precise measure of ageism. Canadian Journal on Aging/la Revue Canadienne du Vieillissement 9: 56-66.

13. Kogan N (1961) Attitudes toward old people: The development of a scale and an examination of correlates. J Abnorm Soc Psychol 62: 44-54.

14. Rupp DE, Vodanovich SJ, Credé M (2005) The multidimensional nature of ageism: Construct validity and group differences. J Soc Psychol 145: 335 362

15. Leung S, Logiudice D, Schwarz J, Brand C (2011) Hospital doctors' attitudes towards older people. Intern Med J 41: 308-314.

16. Bodner E, Bergman YS, Cohen-Fridel S (2012) Different dimensions of ageist attitudes among men and women: A multigenerational perspective. Int Psychogeriatr 24: 895-901.

17. Rodgers V, Gilmour J (2011) Shaping student nurses' attitudes towards older people through learning and experience. Nurs Prax NZ 27: 13-20.

18. Doherty M, Mitchell EA, O'Neill S (2011) Attitudes of healthcare workers towards older people in a rural population: A survey using the Kogan scale. Nursing Research and Practice.

19. Söderhamn O, Gustavsson SM, Lindencrona C (2000) Reliability and validity of a Swedish version of Kogan's old people scale. Scand J Caring Sci 14: 211-215.

20. Vitman-Schorr A, Iecovich E, Alfasi N (2013) Reliability and validity of a Hebrew version of the Kogan's attitudes toward old people scale. Educational Gerontology 40: 315-326.

21. Narita K, Shimonaka J, Nakazato K (1993) Application of Self-Consciousness Scale to the older people - comparison with the youth group. Japanese Journal of Gerontology 37: 48-57.

22. Shimizu H (2010) The problems and characteristics of communication between nursing students and the elderly. Journal of Japan Academy of Gerontological Nursing 11: 56-63.

23. Shimizu H (2010) Validity and reliability of Dialogue Preference Scales for Elders. Journal of Japan Academy of Gerontological Nursing 14: 34-41.

24. Gekoski W, Knox V (1990) Ageism or healthism: Perceptions based on age and health Status. Journal of Aging and Health 2: 15-27.

25. Mori I, Tanno Y (2001) Reliability and validity of creating Social Anxiety Scale in Social Situations scale. Journal of Health Research 14: 23-31.

26. Shankar KN, Bhatia BK, Schuur JD (2014) Toward patient-centered care: A systematic review of older adults' views of quality emergency care. Ann Emerg Med 63: 529-550.

27. Shimizu H, Nojiri M (2005) Student training for better communication with elderly using simulated patients. Journal of Human Care Studies 6: 45-54.

28. Zisberg A, Band-Wintershtein T, Topaz M (2011) Nurses attitudes toward older adults: A comparison between freshmen nursing students and young nurses. In L Zysberg, Student Attitudes. Nova Publisher.
29. Caris-Verhallen WM, de Gruijter IM, Kerkstra A, Bensing JM (1999) Factors related to nurse communication with elderly people. J Adv Nurs 30: 11061117.

30. Rippon I, Kneale D, de Oliveira C, Demakakos P, Steptoe A, et al. (2014) Perceived age discrimination in older adults. Age and Ageing 43: 379-386.

31. Usta YY, Demir Y, Yönder M, Yildiz A (2012) Nursing students' attitudes toward ageism in Turkey. Arch Gerontol Geriatr 54: 90-93.

32. Skela-Savič B, Hvalič Touzery S (2014) Znanja in stališča študentov zdravstvene nege in kliničnih mentorjev do dela s starostniki. Zdravniski Vestnik 83: 102-114. 